\title{
Assessment of Sperm Quality - A Light Microscope Study
}

\author{
Jyothi A. Raj ${ }^{1}$, Heera Sankar², Sagarika Mahapatra 3 , Ashima Binny ${ }^{4}$ \\ 1,2,3,4 Department of Pathology, Rajarajeswari Medical College and Hospital, \\ Mysore Road, Bangalore, Karnataka, India.
}

\section{ABSTRACT}

\section{BACKGROUND}

Semen analysis is an integral part of work up for infertility in men, with sperm morphology being an important qualitative parameter. Qualitative defects can affect any part of the sperm and are classified as defects in the head, middle piece, and tail, based on morphology. The focus of the study was to assess qualitative defects in sperms by light microscopy, in semen with normal sperm counts.

\section{METHODS}

This study is hospital based, descriptive, retrospective study. Of the semen samples received in the clinical laboratory, fifty with normal sperm counts were included in the study and processed according to standard protocol. For evaluation of qualitative defects by sperm morphology, smears were fixed in ethanol, stained with Papanicolaou stain [PAP], and assessed under light microscope.

\section{RESULTS}

The 50 semen samples included in the study had sperm counts ranging from 15 to 80 million / ml. Thirty samples had less than $10 \%$ abnormal forms, fourteen samples had 11 - $20 \%$ abnormal forms, five samples had 21 - $30 \%$ abnormal forms and one sample had $40 \%$ abnormal sperms. Qualitative defects were classified as morphological abnormalities in head, neck, and tail. Of the fifty cases, most defects were found in the head, followed by those in the neck and tail. Common defects noted were double head (44\%), abnormal sized heads, and bent neck (48\%). Coiling was a common defect noted in the tail (10\%). Most sperms showed a combination of defects.

\section{CONCLUSIONS}

Qualitative defects in sperm morphology are often seen in samples with normal sperm counts. Assessment of microscopic characteristics of human spermatozoa is as important as count and motility in the complete evaluation and work-up of semen samples in cases of infertility.

\section{KEY WORDS}

Semen, Sperm, Quality, Microscopy, Morphology
Corresponding Author: Dr. Jyothi A. Raj, Rajarajeswari Medical College and Hospital, Mysore Road, Bangalore- 560074, Karnataka, India. E-mail: jyoki255@gmail.com

DOI: $10.14260 / \mathrm{jemds} / 2021 / 299$

How to Cite This Article:

Raj JA, Sankar H, Mahapatra S, et al. Assessment of sperm quality - a light microscope study. J Evolution Med Dent Sci 2021;10(19):1417-1421, 10.14260/jemds/2021/299 DOI:

Submission 24-10-2020,

Peer Review 11-03-2021,

Acceptance 17-03-2021,

Published 10-05-2021.

Copyright () 2021 Jyothi A. Raj et al. This is an open access article distributed under Creative Commons Attribution License [Attribution 4.0 International (CC BY 4.0)] 


\section{BACKGROUND}

Infertility affects $15 \%$ of couples globally, of which 20 - $30 \%$ are due to defects in sperms. ${ }^{1}$ Semen analysis is an important and routine investigation in the workup for infertility in men and is done using the World Health Organization (WHO) criteria for quantitative and qualitative examination. Qualitative examination of semen is a vital parameter and includes assessment of morphology by microscopic examination of sperms. Morphologic features of sperm is the result of a highly complex process of cellular modifications occurring during spermatogenesis. ${ }^{2}$ A sperm consists of a head, neck, middle piece (midpiece), principal piece and endpiece. The endpiece is difficult to see with a light microscope, so sperms are considered to comprise head (and neck) and tail (midpiece and principal piece). For a spermatozoon to be considered normal, both its head and tail must be normal. All borderline forms should be considered abnormal. ${ }^{3}$ Morphological defects can be found in any or all of these parts. Accordingly, sperm defects are categorised as those in head, middle piece, and tail. ${ }^{1}$ Evaluation of sperm morphology and quality is confusing and time-consuming. The difficulty in assessment is due to lack of objectivity, variation in interpretation or poor performance. WHO recommends a simple normal / abnormal classification, tallying the location of abnormalities in abnormal spermatozoa. Studies have shown that the percentage of normal spermatozoa and the mean number of abnormalities per spermatozoa correlates more closely with the fertilization rate than sperm count and motility. Environmental and lifestyle factors such as smoking and alcohol use are known to affect the sperm morphology and are associated with specific abnormalities. ${ }^{2}$ Defects due to stress or medication are reversible, whereas those due to genetic defects are severe and incapable of fertilization. ${ }^{2}$

An increased percentage of spermatozoa with abnormal shapes is commonly associated with defective spermatogenesis. Fertilizing potential of abnormal spermatozoa decreases, depending on the types of anomalies. They may also have abnormal deoxyribonucleic acid (DNA). ${ }^{3}$ Successful fertilization and early embryonic development in assisted reproductive techniques depends on the morphology of spermatozoa. Sperm morphology is better assessed by staining, using one of the many staining methods such as Papanicolaou (PAP), Haematoxylin-Eosin, Giemsa, and DiffQuik stains. Papanicolaou stain is one of the preferred methods for evaluating sperm morphology and quality in routine laboratory practice. Different staining methods may cause some changes in the morphometric values of spermatozoa because fixatives can induce slight cell shrinkage. Some authors recommend the use of combination of stains to overcome this limitation for morphometric measurements.3,4

The process of slide smear preparation from the semen sample is time consuming. Assessment of sperm morphology requires semen smear preparations to be of high quality. Small artefacts also might influence the appearance of the sperm. Technique dependent source of errors can be minimized with standardized and controlled methods. Good quality smears depend on the quantity of the stain used, time allowed for the mixture to stand and preparation of the smear. Certain precautions like use of minimal force with slides while making smears manually help prevent broken tails. The slides should be preferably cleaned with $95 \%$ or absolute alcohol before use. ${ }^{5}$

\section{Objectives}

This study intends to assess qualitative defects in sperms by light microscopy, in semen with normal sperm counts, in men attending infertility clinic.

\section{METHODS}

This is a hospital based, descriptive, retrospective study conducted in the Department of Pathology, Rajarajeswari Medical College and Hospital, Bangalore. A total of 80 semen samples were received over a period of six months from January 2018 to June 2018, of which 50 samples with normal sperm counts were included in the study and evaluated for qualitative defects in sperms. The study was approved by ethics committee and informed consent was obtained. Details were recorded in standard proforma and samples processed according to protocol as follows:

Samples were collected in a clean-capped plastic container and assessed when fresh

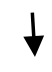

After liquefaction, volume, colour, appearance and viscosity were noted

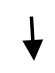

For sperm quality, smears were fixed in ethanol and stained with Papanicolaou stain

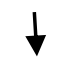

On microscopy, sperm count, motility, agglutination and sperm morphology were documented

\section{Count ${ }^{3}$}

Wait for liquefaction

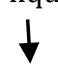

Mix $1 \mathrm{ml}$ liquefied semen with $20 \mathrm{ml}$ diluting fluid (Sodium Bicarbonate - formalin)

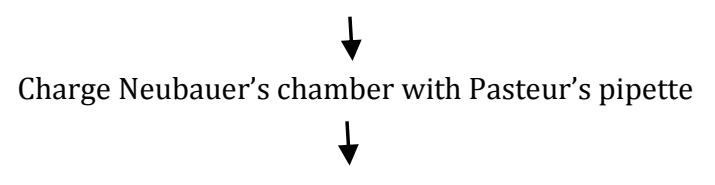

Place chamber in humid conditions for 10 - 15 min

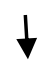

Count in four large corners

$$
\begin{aligned}
& \text { Calculation } \\
& \text { Count }(/ \mathrm{ml})=\frac{\text { sperms counted } \times \text { correction for dilution } \times 1000}{\text { No. of squares counted } \times \text { Volume of one square }} \\
& =\mathrm{N} \times 20 \times \frac{1000}{4 \times 0.1} \\
& =\mathrm{N} \times 50000
\end{aligned}
$$




\section{Morphology}

Place a drop of seminal fluid on slide

$$
\downarrow
$$

Stain with Papanicolaou stain

\section{$\downarrow$}

Look for morphology of at least 200 sperms

\section{Inclusion Criteria}

All semen samples with normal sperm counts.

\section{Exclusion Criteria}

Semen samples diluted with urine and those with abnormal counts.

\section{Statistical Analysis}

Detailed data was recorded in MS Excel and statistically analysed using IBM SPSS v20 software. Data was expressed in numbers, percentage, and tables.

\section{RESULTS}

\begin{tabular}{|c|c|c|c|}
\hline Part of Sperm & Defects & Number of Cases & Percentage \\
\hline \multirow{7}{*}{ Head } & Double & 22 & 44 \\
\hline & Large & 17 & 34 \\
\hline & Small & 17 & 34 \\
\hline & Round & 6 & 12 \\
\hline & Globular & 3 & 6 \\
\hline & Tapering & 2 & 4 \\
\hline & Others & 8 & 16 \\
\hline \multicolumn{4}{|c|}{ Table 1. Qualitative Defects in Sperm Heads } \\
\hline
\end{tabular}

\begin{tabular}{|cccc|}
\hline Part of Sperm & Defects & Number of Cases & Percentage \\
\cline { 2 - 4 } Middle piece & Bent neck & 24 & $48 \%$ \\
& Short neck & 2 & $4 \%$ \\
& Thick midpiece & 1 & $2 \%$ \\
\hline \multicolumn{2}{|c|}{ Table 2. Qualitative Defects in Middle Piece of Sperms } \\
\hline
\end{tabular}

The 50 semen samples included in the study had sperm counts ranging from 15 to 80 million / ml. Approximately 200 spermatozoa in each sample were evaluated for normal and abnormal forms. Thirty samples had less than $10 \%$ abnormal forms, fourteen samples had $11-20 \%$ abnormal, five samples had 21 - $30 \%$ abnormal, and one sample had $40 \%$ abnormal sperms. Various morphological defects found in these samples were documented as follows -

- Common defects in the head were double head [22 cases$44 \%$ ], followed by large and small heads in 17 cases each [Table 1].

- Middle piece defects were most common followed by those in the head and tail [Tables $2 \& 3$ ].

- Most common defect in the middle piece was bent neck [Table 2].

- 35 cases had sperms with more than one defect [Table 4].

- Head and neck abnormalities were the most frequent combinations followed by head and tail abnormalities.

- Seven cases had sperms multiple defects in the head, neck, and tail.
- The morphological defects are depicted in figures [1 to 6].

\begin{tabular}{|cccc|}
\hline Part of Sperm & Defects & Number of Cases & Percentage \\
& Coiled & 5 & $10 \%$ \\
Tail & Double & 4 & $8 \%$ \\
& Short & 3 & $6 \%$ \\
& Absent & 2 & $4 \%$ \\
\hline \multicolumn{3}{|c}{ Table 3. Qualitative Defects in Sperm Tails } \\
\hline
\end{tabular}

\begin{tabular}{|ccc|}
\hline $\begin{array}{c}\text { Combination of } \\
\text { Defects }\end{array}$ & $\begin{array}{c}\text { Number of Cases with } \\
\text { Multiple Defects in Sperms }\end{array}$ & Percentage \\
Head and neck & 20 & $40 \%$ \\
Head and tail & 12 & $24 \%$ \\
Head and middle piece & 2 & $4 \%$ \\
Neck and tail & 9 & $18 \%$ \\
Head, neck, and tail & 7 & $14 \%$ \\
\hline \multicolumn{2}{|c}{ Table 4. Multiple Qualitative Defects } \\
\hline
\end{tabular}

\section{DISCUSSION}

Of the total population, approximately fifteen percent of married couples are having infertility, twenty percent is thought to originate from men. ${ }^{4}$ According to World Health Organisation estimate, the overall prevalence of primary infertility in India is between 3.9 and $16.8 \%$ [2016].

Assessment of sperm morphology is an important parameter in the work-up of infertile men. Due to increasing use of in vitro fertilization techniques, studies are being focused on the role of sperm morphology in fertilization. Sperm quality is important for successful fertilization and early embryonic development in assisted reproductive techniques. ${ }^{4}$ It is considered to be one of the best discriminators for fertilization potential. ${ }^{3}$ Until the 20th century, little attention was given to assessment of sperm morphology. ${ }^{5}$ There was also lack of uniformity in evaluation of morphology. In 1999, World Health Organisation published guidelines for semen analysis. Fourth and fifth editions of WHO manual are the most recommended by fertility physicians. $^{2}$

Qualitative defects are assessed by microscopic examination of sperm morphology and are an important parameter in semen analysis. Head, middle piece, and tail form parts of a sperm. Each of these parts can present with different morphological abnormalities.

Studies have shown that some of these defects are irreversible, while those due to acquired/environmental factors can be reversible. ${ }^{6}$ Lifestyle factors such as smoking and alcohol use, are thought to affect the morphologic features of sperm. ${ }^{2}$

Evaluation is done by staining spermatozoa from fresh semen and examination under light microscope. Morphology of sperms can be assessed better when they are stained and Papanicolaou stain gives good staining of spermatozoa. 3,4 Aksoy $\mathrm{E}$ et al. $^{4}$ used different staining methods to assess morphometric measurements and morphology of spermatozoa under light microscopy, in 67 patients. They found changes in the morphometric values of spermatozoa because the fixatives induced slight cell shrinkage. They concluded that for morphological assessment of spermatozoa, Papanicolaou, Haematoxylin-Eosin (HE), toluidine blue and Shorr stains are the best dyes for staining quality. 


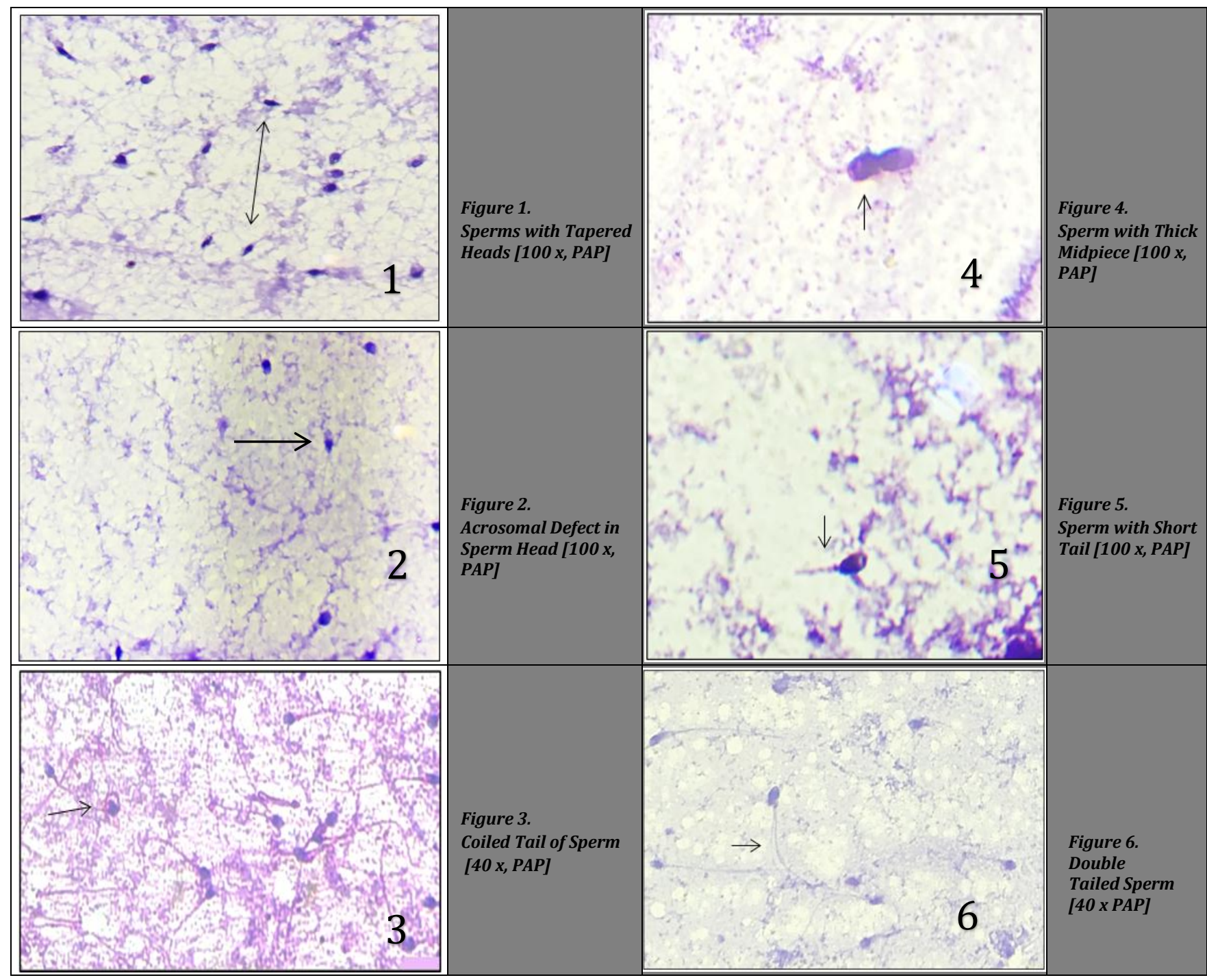

Tygerberg Classification Criteria described by Kruger [1986] and the WHO classification are the most important morphological classifications of spermatozoa. Spermatozoa consist of a head, neck, middle piece (midpiece), principal piece and endpiece. With a light microscope, the cell can be considered to comprise a head (and neck) and tail (midpiece and principal piece). ${ }^{3}$

For a spermatozoon to be considered normal, both its head and tail must be normal. All borderline forms should be considered abnormal. According to Kruger, in a normal sperm, the boundaries of the head should be smooth, regularly contoured and oval. There should be a well-defined acrosomal region coating $40-70 \%$ of the head area. The acrosomal region should contain no large vacuoles. Sperm acrosome size and staining abnormality is one of the important criteria identified for sperm morphology evaluation based on sperm functionality. ${ }^{6}$ Midpiece should be slender, long, regular and about the same or 1.5 times the length as the sperm head, with the axis of the midpiece aligned with the axis of the sperm head. The principal piece or tail should be thinner than middle piece and should have a uniform calibre along its length. It should be flat, without wrinkles, and not contain broken parts and cytoplasmic debris. It should be approximately $45 \mu \mathrm{m}$ long (about 10 times the head length).3,4,7

Most morphological abnormalities occur in combination. Multiple defects cause defective development of embryo and are associated with increased chances of spontaneous abortions. Morphologically abnormal spermatozoa and semen leucocytes generate reactive oxygen species, which may damage sperm structure, leading to reduced motility and DNA damage. This may interfere with early embryo development, resulting in spontaneous abortions. ${ }^{1,8}$

The fifth edition of WHO manual proposes a very low cutoff value of $4 \%$ for morphologically normal spermatozoa. On its own, this value may not provide a strong predictive value for a male's fertility potential. The same can however be obtained with a holistic strict approach for sperm morphology evaluation with additional morphology parameters. ${ }^{6}$

Three indices can be derived from the detailed assessment of morphological abnormalities of the head, midpiece and principal piece: the multiple anomalies index (MAI), the teratozoospermia index (TZI), and the sperm deformity index (SDI). These indices have been correlated with fertility in vivo (MAI and TZI) and in vitro (SDI) in various studies and may be useful in assessment of exposures or pathological conditions. A TZI of 1.6 or more is associated with a lower pregnancy rate, and an SDI value of 1.6 or more is the cut-off for failure of in vitro fertilization. These indices can only be derived by using the manual method.2, 3, and 7,9,10

Menkveld $\mathrm{R}$ et al. have described defects such as tapering and megaloheads to be reversible, possibly induced by stress or medication, and revert on withdrawal of precipitating 
factor. Others such as head-neck attachment, misaligned neck, short tail syndrome or abnormal neck insertions are genetically determined and have poor prognosis. ${ }^{1,11}$

Defects in the head are the most common and include tapering, large, round, short, amorphous, and bifid forms. Defects such as amorphous head and globozoospermia are genetically determined. Goyal $\mathrm{R}$ et al. described tapering head to be the most common defect, followed by middle piece defects. ${ }^{1}$ In the present study, midpiece defects were more common.

Neck defects such as bent neck is genetic and carry poor prognosis. Cytoplasmic residue or excess is associated with sperm immaturity and production of reactive oxygen species implying ongoing stress. ${ }^{7}$

Short tail syndrome is genetically determined and has very poor chance of future fertility. Coiled tails result in defective propulsion. Tail abnormalities are increased in smokers. Coiled tail was the least common abnormality detected in the study by Goyal et al. ${ }^{1}$ In the present study too, tail abnormalities were least common, with coiled tails seen in 10 $\%$ of cases.

Table 5 below shows the reference range and lower reference limit of various parameters in a sample of semen, derived from the WHO laboratory manual for examination and processing of human semen. $\left[2010 ; 5^{\text {th }} \text { edition }\right]^{3}$

\begin{tabular}{|c|c|c|}
\hline & Parameter & Lower Reference Limit \\
\hline & Semen volume (ml) & $1.5(1.4-1.7)$ \\
\hline & Total sperm number $\left(10^{6}\right.$ per ejaculate $)$ & $39(33-46)$ \\
\hline & Sperm concentration $\left(10^{6}\right.$ per ml $)$ & $15(12-16)$ \\
\hline & Total motility (PR + NP, \%) & $40(38-42)$ \\
\hline & Progressive motility (PR, \%) & $32(31-34)$ \\
\hline & Vitality (live spermatozoa, \%) & $58(55-63)$ \\
\hline & Sperm morphology (normal forms, \%) & $4(3.0-4.0)$ \\
\hline \multirow{7}{*}{$\begin{array}{l}\text { Other } \\
\text { consensus } \\
\text { threshold } \\
\text { values }\end{array}$} & $\mathrm{PH}$ & $\geq 7.2$ \\
\hline & $\begin{array}{l}\text { Peroxidase - positive leukocytes }\left(10^{6}\right. \\
\text { per ml })\end{array}$ & $<1.0$ \\
\hline & $\begin{array}{l}\text { MAR test (motile spermatozoa with } \\
\text { bound particles, \%) }\end{array}$ & $<50$ \\
\hline & $\begin{array}{l}\text { Immunobead test (motile spermatozoa } \\
\text { with bound particles, \%) }\end{array}$ & $<50$ \\
\hline & Seminal zinc ( $\mu \mathrm{mol}$ / ejaculate) & $\geq 2.4$ \\
\hline & Seminal fructose ( $\mu \mathrm{mol}$ / ejaculate) & $\geq 13$ \\
\hline & $\begin{array}{l}\text { Seminal neutral glucosidase }(\mathrm{mU} / \\
\text { ejaculate) }\end{array}$ & $\geq 20$ \\
\hline \multicolumn{3}{|c|}{$\begin{array}{l}\text { Table 5. Lower Reference Limits ( } 5^{\text {th }} \text { Centiles and Their } 95 \% \\
\text { Confidence Intervals) for Semen Characteristics [WHO] }\end{array}$} \\
\hline
\end{tabular}

\section{CONCLUSIONS}

Quality of spermatozoa has a direct influence on fertilization and developmental competence of embryos. Cytomorphologic analysis of sperm quality by light microscopy is a useful initial screening test for the evaluation of sperm. It helps clinicians in making decisions for in vitro fertilization. Lifestyle modifications may be recommended as a measure to improve sperm morphology in patients with fertility problems.
Data sharing statement provided by the authors is available with the full text of this article at jemds.com.

Financial or other competing interests: None.

Disclosure forms provided by the authors are available with the full text of this article at jemds.com.

\section{REFERENCES}

[1] Goyal R, Kotru M, Gogia A, et al. Qualitative defects with normal sperm counts in a patient attending infertility clinic. Indian J Pathol Microbiol 2018;61(2):233-5.

[2] Singh S, Sharma S, Jain $M$, et al. Importance of papanicolaou staining for sperm morphologic analysis: comparison with an automated sperm quality analyzer. Am J Clin Pathol 2011;136(2):247-51.

[3] Cooper TG. WHO laboratory manual for examination and processing of human semen. $5^{\text {th }}$ edn. Geneva, Switzerland: WHO Press 2010.

[4] Aksoy E, Aktan TM, Duman S, et al. Assessment of spermatozoa morphology under light microscopy with different histologic stains and comparison of morphometric measurements. Int $\mathrm{J}$ Morphol 2012;30(4):1544-50.

[5] Victor R, Sebastian D, Lakshmi TA, et al. A Pilot study for a simple, rapid direct staining technique for human sperm morphology. International Journal of Health Sciences and Research 2014;4(4):116-9.

[6] Menkveld R. Clinical significance of the low normal sperm morphology value as proposed in the fifth edition of the WHO laboratory manual for the examination and processing of human semen. Asian J Androl 2010;12(1):47-58.

[7] Menkveld R, Wong WY, Lombard CJ, et al. Semen parameters, including WHO and strict criteria morphology, in a fertile and subfertile population: an effort towards standardization of in-vivo thresholds. Hum Reprod 2001;16(6):1165-71.

[8] Cao X, Cui Y, Zhang X, et al. The correlation of sperm morphology with unexplained recurrent spontaneous abortion: a systematic review and meta-analysis. Oncotarget 2017;8(33):55646-56.

[9] Auger J, Eustache F, Anderson AG, et al. Sperm morphological defects related to environment, lifestyle and medical history of 1001 male partners of pregnant women from four European cities. Hum Reprod 2001;16(12):2710-7.

[10] Aziz N, Saleh RA, Sharma RK, et al. Novel association between sperm reactive oxygen species production, sperm morphological defects and the sperm deformity index. Fertil Steril 2004;81(2):349-54.

[11] Menkveld R, Stander FS, Kotze TJ, et al. The evaluation of morphological characteristics of human spermatozoa according to stricter criteria. Hum Reprod 1990;5(5):586-92. 